\title{
Balantidiasis en el niño, presentación de un caso clínico
}

Muraleda L.", Díaz G. ${ }^{* *}$, Isracl E.***

\begin{abstract}
The clinical course of a 10 years old boy wilb a lnteatinal Balantidiasis and a Trichocephalosis is presented. Because the few reports about that intestinal protozos in our country, it is very importent, consider this poseibility in the differential diagnosis of dysenteric syndrom, specially in patients from suburban areas.
\end{abstract}

Balantidiasis constituye una Zoonosis, cuya incidencia en el hombre pareciera ser baja, estando por lo general asociada a una deficiente sanidad del medio y desnutrición. ' " Se ha descrito una mayor prevalencia en aquellas zonas rurales en donde la crianza de cerdos̀ escapa a un adecuado control sanitario. ${ }^{2}$

De los casos de Balantidiasis humana la mayor parte compromete a sujetos adultos. (3) Ello probablemente está en relación con ciertas actividades laborales y proximidad a la fuente infectante. 12 :

El tracto digestivo aparece como el más frecuentemente comprometido, no obstante se ha descrito en humanos el hallazgo de Balantidium coli en líquido cefalorraquided ${ }^{(4)}$ y mucus cérvico uterino. ${ }^{(5)}$

Consideramos de interés presentar esta comunicación clínica con el fin de hacer una contribución a la bibliografia existente del tema y a la vez hacer presente al accionar clínico y diagnóstico la posibilidad de una Balantidiasis en la etiología de un Sindrome disentérico.

\section{CASO CLINICO:}

J. 1.T. de 10 años de edad. sexu masculino, presedente de zona rural situgda a $20 \mathrm{Km}$. de Máfil, provincia de Valdivia. Entre sus antecedenlfa Hestaca ser portador de desnutrición severa ya evidenciada an ferímda de lactunte menor.

*: Institulı de Pediatríu. Institutu de Parasitulogia; raculsad de Medicitu L A.C.b.

**: Tecnólogo Medico Hospital Regional Valdivia

***: Interno de Mrdirina, Facultad de Medicina, U.A.Ch.
Su enfermedad actual se inicia un mes previo a su ingrese con compromiso progresivo del estado general, fiebre, alteración del tránsito intestinal cararterizado par periodus de constipaciour alternados con episndios de deposiciones diarreicas con caráter disentirica. Al ingress del paciente al Serviciu de Pediatria del Hospial Kegional de Valdivia, se descrihe en regulares condiciunex senerales. febril, ron retraso globat del denarrollo y marcado retardo mental, peso 113 . 300 y talla (101 cms.) hajo el percentil 3, abdomen prominente sin visceromegulia. El resto del examen revela una serie de hallazgos genotipicos. compatiblez cun una zenopatia, probablemente un Sindrome de Noonan.

Durante su evolución intrahuspitalaria se evidencla un Sindrome disentérico, cuya rvaluación etiológica por medio de cxamen parasitclugicu (Teleman modificado), con 3 muestras seriadas revedi presencia de numerosos trofozoides de Bciantiditus coli y huevos de Trichuris Trichuria, siendo negativo desde el punto de vista bacteriolígico. Oımos exámenes reglizados en forma simultánea permitieron descartar un Síndrone de Malabsorcicin. revelaron parámetros hematolúgicos y nuveles séricos de inmunuglobulinas normales. Enduscopía mustró signus inflanaiurius inespecificos en mucosa rectul.

El paciente fue tratado con Tetraciclinu durante 10 dias. posterior a lo cual se realizé terapia contra el iricucefolo con Mebendazole. Al lercet dis de tratamienın anlibiótico se evidenció normalizacisin de deposicjones y mejoria del catudo general. Los controles parasitolicicos fueron negativos.

Respec los al estudio epidemiológicu, es necesarin destacar que J.A.T. es un paciente que por su estado de salud ba vivido confingdo en su casa. Su grupo fomiliar está constituido por 13 personas que viven hacinadas, en malas condiciunes higiénicas y cuya única fuente de agua proviene de una vertiente subterrínea gue por su disposición bu es probable au cantaminación con depusieivines de animales $v$ exiretog humanos.

En busta de una fuente de infeccion intrafamiliar, se prucedio a realizar exdmenes parasituligicury yeriados (3 muesteas). pur las mítudes PAFS \& MTM. a la titalidad del grupo familiar. sjendo '́stuss negutivos para el protuzoo. Idénticus resultadou se nbluvierun en el estudio parasitologico de low animales doméstiros prixinus a la vivienda. Antesedente rejevante lo constituye la ausencia de cerdos en una exlensa área circundante al grupo familiar en hos tiltinutes 4 anios. 


\section{COMENTARIO}

Se presentan los anteredentes clínicos y epidemioligicos de un paciente de 10 años de edad, portador de un Síndrome disentéricu, en el cual la investigación parasitológica de deposiciones permite confirmar el diagnóstico de Balantidiasis intestinal.

Diferentes autores coinciden en señalar que la presencia de Balantidium coli en cerdos es elevadat l. 2. '́, no obstante la presencia de este protozoo, ciliado como agente etiológico de Síndromes disentéricos en el hombre pareciera ser haja.

Estudios locales respecto al hallazgo de Balantidium coli en cerdos revelan que el $83,7 \%$ de estus animales son purtadores del parásito. Experimentalmente se ha demostrado que la capacidad de este protozoo para multiplicarse $y$ producir alteraciones en el intestino de un huésped extraño varía enormemente(8), lo cual pudiera explicar la baja incidencia de esta infeccion en el hombre, aun en medios altamente contaminados.

La desnutrición y la presencia de otros agentes patógenos en el intestino parecieran constituir factores condicionantes que favorecen y acrecientan la patogenicidad del $\boldsymbol{B}$, coli.1, hl El paciente que motiva esta presentación reúne estas dos condicior nantes.

En lo referente a la fuente de contagio. los datos ubtenidos en el estudio epidemiológico nos aportan a)gunos elementos indirectos que nus permiten formular una hipitesis. Creemos que podemos negar con cierta certeza la posibilidad de comtagja intrafamiliar actual, debido a que las técnicas usadas para el estudio del $B$ coli en el grupo familiar nos dan sobre el $90 \%$ de protuabiliedad de hallazgo del parásito. 18। En base a lo anterior y al antecedente de que el paciente poseid una movilidad limitada, existe la posibilidad de un contazio de hace : por to menos 4 años, manteniendo al paciente por su desnutrición, en un estado de portador erónico. (un manifestaciones clínicas reasionales, siendo el episodio disentérico actual el de mayor importancia. Eklas condicimantes no se presentan en el resto de la lamilia.
El cuadro clinico de este paciente corresponde a la forma de balantidiasicrínica reagudizada. $21 \mathrm{La}$ respuesta al tratamiento con Tetraciclina nos parece satisfactoria en términus de regresión de sintomatología y negativizarión de hallazgos parasitoligicos, lo cual concuerda con otras comunicaciones. 110 .

\section{RESUMEN}

Se presenta el casu clinico de un paciente de 10 años de edad, el cual es portador de un cuadro do Balontidiasis intestinales asoviado a Tricucefalosis. Dada las escasas comunicaciunes en uuestro medio de esta Protozoosis intestinal, se hace mención a la literatura publicada y se destacia la importancia de pensar en esta etiología en pacientes portadores de Sindromes disentéricos, especialmente cuandos som de prescedencia rural.

\section{REFERENCLAS}

I Fuust, E. C.; Russell, P. F, and Jung, R. C. "Parasitología Clinica". Edit, Satvut, 1974.

${ }^{2}$ Attas, A., Neghme, A. "Parasitolugia Clinica". Edit. Intermedia. Buenos Aires, 1979.

3 Lembreras, H. "Balantidiasis en el Perú". Rev, Médica Peruana, 25: 463-531, 1954.

4 Seamster, A. "An unusual ciliate infection of the spital fluid". The Texas Journal of Science. December 30, 1952, N." 4, pp. $531-332$.

5 James, G.; Noman and Pamela Jessop. "Balantidium coli in a cervice smear." Am. Jour. Australia, 1: 644, 1973.

6 Levilte, N. "Proluzoan Parasites of Domeatic Animals and Man." Secund Edition, Burgess Publishing Company, Minneapelis. USA, 1973.

7 Letonia, T.; Henrtquez, A,; Heyes, G.; Zayata, L. "Prevalencia de infecciones por Balantidium coli en cuerdos de Santiago, Chile." Bol. Chile Parasit., 30: 86-89. 1975.

8 Gabatdon, A. "Balantidium coli: Quantilative studies in experimental infections and varialiugs in infectiousness for rats." J. Parasitol. 21: 336-392, 1935.

${ }^{9}$ Neghme', A., te al. "Contribucion a la quimióterapiy del Balaslidium coli. I. Estudio 'in vitro'." Biológica [Sanliago\}, J2-13: 109-116. 1951.

10 Naduiru, F. y colaboradores. "Cuatru casos de Balantidiasis humana." Bul. Chile Parasit. J7: 49. 1962.

11 Hauser, M.; Santibeinez, J. M. "F'revalencia de Las infecciones por prutozoos y helmintus intestinales del hombre y agouas axunosis de perrus y cerdos en el sector ribereño del lago Calarquén. en relación a las condiciones del saneamientu ambiental." Tesis. Faculad de Medicina. Escuela Tecnologis Médica Unirersidad de Chile, Yaldivia, 1978; 